\title{
Photophysical Properties of Benzophenone Based TADF Emitters in Relation to Their Molecular Structure
}

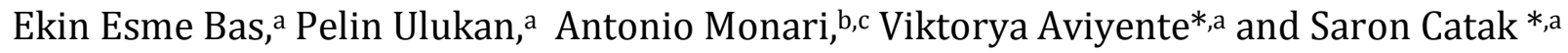 \\ aDepartment of Chemistry, Bogazici University, Bebek, 34342 Istanbul, Turkey \\ bUniversité de Lorraine and CNRS, LPCT UMR 7019, F54000 Nancy, France \\ cUniversité de Paris and CNRS, ITODYS, F75006 Paris, France
}

\begin{abstract}
Thermally activated delayed fluorescence (TADF) materials are commonly used in various apparatus, including organic light emitting device (OLED)-based displays, as they remarkably improve the internal quantum efficiencies (IQE). Although there is a wide range of donor-acceptor based compounds possessing TADF properties, in this computational study we investigated TADF and some non-TADF chromophores, containing benzophenone or its structural derivatives as the acceptor core, together with various donor moieties. Following the computational modeling of the emitters, several excited state properties, such as the absorption spectra, singlet-triplet energy gaps $\left(\Delta \mathrm{E}_{\mathrm{ST}}\right)$, natural transition orbitals (NTO) and the topological $\Phi_{\mathrm{s}}$ indices, have been computed. Along with the donor-acceptor torsion angles and spin-orbit coupling (SOC) values, these descriptors have been utilized to investigate potential TADF efficiency. Our study has shown that on the one hand, our photophysical/structural descriptors and computational methodologies predict the experimental results quite well, on the other hand, our extensive benchmark can be useful to pinpoint the most promising functionals and descriptors for the study of benzophenone based TADF emitters.
\end{abstract}

\section{INTRODUCTION}

Organic Light-Emitting Diodes (OLEDs) have attracted widespread attention since the invention of the first organic electroluminescent (EL) device in $1987 .{ }^{1}$ As compared to conventional light-emitting diodes (LEDs) and liquid crystal display (LCD) systems, OLEDs do not require a backlight unit as they are self-illuminating. Given this distinct feature, OLEDs offer several advantages, such as flexible device structures, decreased panel thickness, improved brightness, and reduced power consumption, rendering them most suitable for various devices. ${ }^{2}$ Nevertheless, OLEDs also display serious efficiency drawbacks, which are grounded in the fundamental spin statistics rule (Figure 1), since the population of the non-emissive triplet state comprises $75 \%$ of the generated excitons in the device, hence leading to the loss of two thirds of the applied energy. ${ }^{3}$ Phosphorescent organic-light emitting diodes (PhOLEDs), which contain heavy atoms can improve the efficiencies of OLEDs with the help of enhanced intersystem crossing (ISC) and improved phosphorescence rates as a result of increased spin-orbit coupling (SOC). ${ }^{4,5}$ However, heavy metals instigate environmental issues while significantly increasing the cost of the device, thus limiting the commercial availability of PhOLEDs. ${ }^{6}$

Thermally activated delayed fluorescence (TADF) materials, first proposed by Adachi et al. in 2011, represent a most suitable alternative to overcome spin statistics burdens and achieve high internal quantum efficiencies (IQE) in OLED displays. ${ }^{7}$ In TADF processes the population of triplet excitons undergoes a slow reverse intersystem crossing (RISC), re-populating the emissive first excited singlet state $\left(\mathrm{S}_{1}\right)$ and hence, avoiding the inclusion of heavy metals. To assure efficiency, RISC or the up-conversion of the triplet states (Up-Intersystem Crossing-UISC) require a small $\mathrm{S}_{1}-\mathrm{T}_{1}$ energy gap $\left(\Delta \mathrm{E}_{\mathrm{ST}}\right)$. UISC can be achieved by promoting the population of intramolecular charge transfer (ICT) states and is usually, albeit not necessarily, correlated with a small gap between the highest occupied molecular orbital (HOMO) and lowest unoccupied molecular orbital (LUMO). To achieve efficient ICT, the molecular architecture requires the presence of a donor (D) moiety bridged to an acceptor (A) (Figure 1). The D and A groups can be separated by using bulky substituents to increase steric effects and maintain orthogonal molecular structures, hence minimizing conjugation and delocalization, or alternatively by $\pi$ bridges. 8,9

Benzophenone is a widely used building block in the design of OLED devices, in part due to its strong and efficient electron accepting and efficient ultraviolet (UV) absorbing abilities, as well as its high ISC efficiency.10-13 Adachi et al. showed in 2014 that efficient deep blue TADF could be achieved by using benzophenone based D-A-D frameworks. ${ }^{12}$ Thus far, many benzophenone based TADF emitters possessing a small $\Delta \mathrm{E}_{S \mathrm{ST}}$ have been reported to feature full-color delayed fluorescence emission, usually in a range 


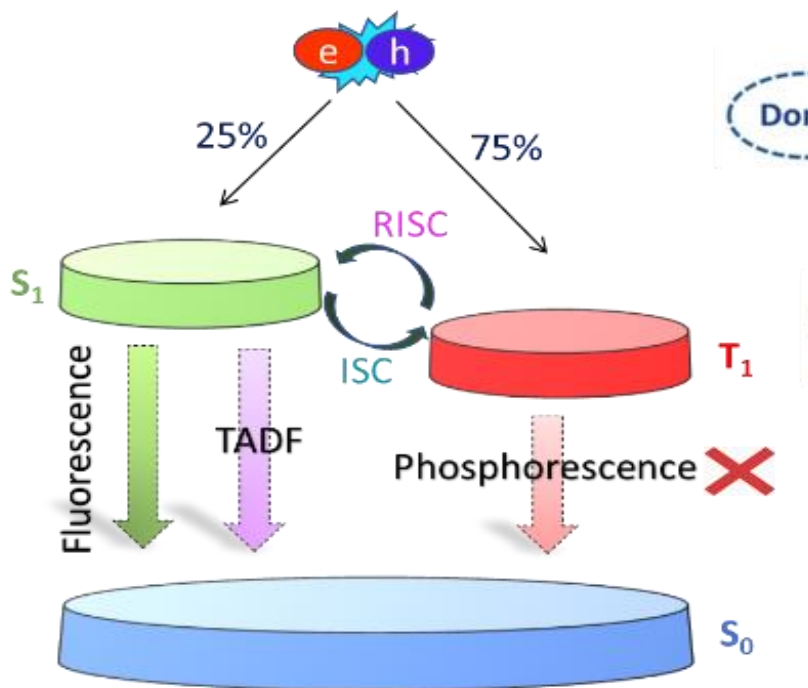

$\underline{\text { TADF Design Strategies }}$

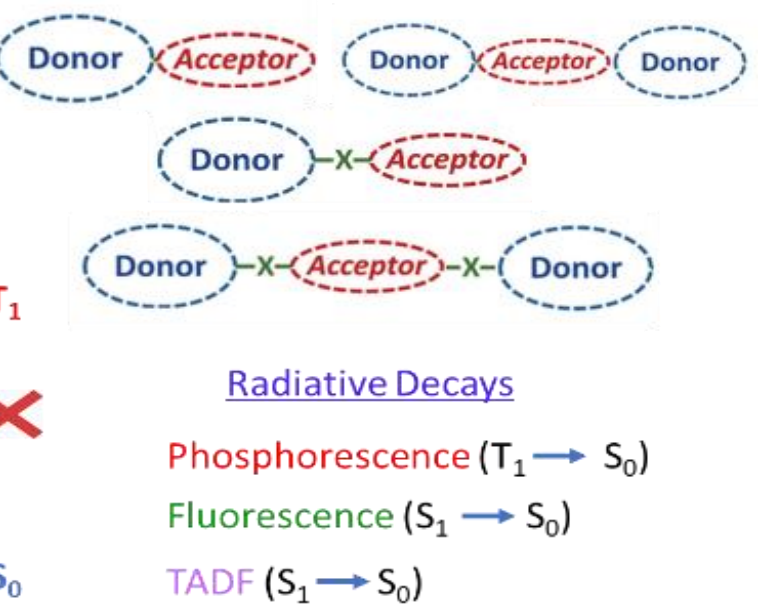

Figure 1. Schematic representation of ISC, RISC, TADF and phosphorescence processes in a Jablonski diagram along with the possible design strategies for TADF emitters.

from deep blue to green and with external quantum efficiencies (EQE) up to $14.3 \% .^{14}$ Benzophenone based luminogens can also be used to induce aggregation-induced delayed fluorescence (AIDF). ${ }^{15,16}$

Nonetheless, the rather floppy phenyl moieties of benzophenone itself may induce intramolecular rotations, which enhance non-radiative decay and lead to relatively low reverse intersystem crossing rates ( $\left.\mathrm{k}_{\mathrm{RISC}}\right) .{ }^{17}$ Hence, more compact and rigid benzophenone derivatives, such as anthraquinone,18,19, xanthone, ${ }^{20-23}$ dibenzothiophene-benzoyl ${ }^{24,25}$ and phenylcarbazole-benzoyl ${ }^{26}$ are deemed more promising in terms of their TADF efficiencies. Figure 2 depicts the molecular structures of benzophenone derivatives commonly used in TADF materials, together with some of the most widely used donor groups.

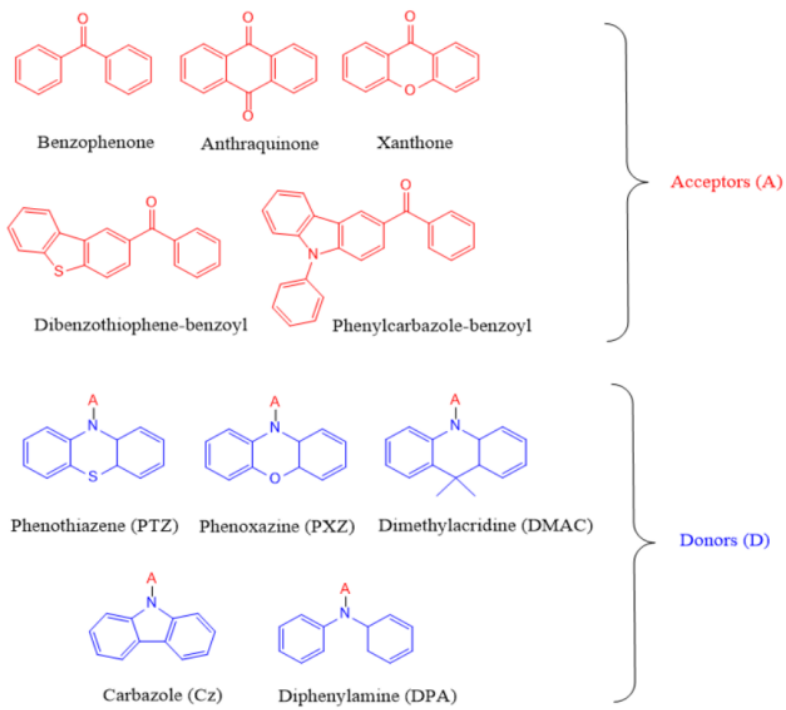

Figure 2. Electron donating and accepting moieties frequently used in the design of benzophenone based TADF emitters.
Molecular modeling is used to elucidate the relationship between molecular structure and optimal photophysical properties that are essential for effective TADF emission. Various descriptors are used to define TADF properties, these include: $\Delta \mathrm{E}_{\mathrm{ST}}$, spin-orbit coupling (SOC), natural transition orbitals (NTOs), the topological $\Phi_{\mathrm{s}}$ index quantifying the charge-transfer amount, and the torsion angles between $\mathrm{D}$ and A moieties. ${ }^{27-33}$ The oscillator strength (f) is also a critical parameter to attain a reasonable radiative decay rate $\left(\mathrm{k}_{\mathrm{r}}\right)$ from the $\mathrm{S}_{1}$ state to the ground state. The oscillator strength is usually closer to 0 for orthogonal donor-acceptor compounds, where charge transfer is prominent. ${ }^{29} \mathrm{Neg}-$ ligible oscillator strengths represent a challenge in TADF emitters, since it is essential to balance a small $\Delta \mathrm{E}_{\text {sт }}$ and a sufficiently large $\mathrm{k}_{\mathrm{r}}$ to reach high internal quantum efficiency (IQE) in OLED devices.

In this study, a series of experimentally studied TADF emitters, employing benzophenone and its derivatives, have been investigated by quantum chemical calculations. Several non-TADF benzophenone chromophores have also been modeled for comparison. Absorption spectra of the selected compounds have been generated, including a sampling of the Franck-Condon region by Wigner distribution, to include dynamic effects. To determine the degree of charge-separation in the excited state, NTOs and $\Phi_{\mathrm{S}}$ indices have been computed, as well as the $\Delta \mathrm{E}_{\mathrm{ST}}$ values. NTO and $\Delta \mathrm{E}_{\mathrm{ST}}$ calculations have been performed on both the ground state $\left(\mathrm{S}_{0}\right)$ and the lowest triplet state $\left(\mathrm{T}_{1}\right)$ equilibrium geometries, since geometrical reorganization can also play a role in photophysical processes. Furthermore, the SOCs between singlet and triplet excited states have been computed to better estimate RISC probability.

The mentioned descriptors have proven to be insightful in determining potential TADF efficiency, as well as shedding light on the correlation between molecular structure and TADF performance. Indeed, the investigation of the 
photphysical characteristics of both the excited and ground state geometries, and also the assessment of the DFT functionals for the excited state calculations of TADF emitters, may help provide a better understanding for the photophysical processes related with TADF at a molecular level.

\section{METHODOLOGY}

All ground state geometry optimizations have been performed using the Gaussian 16 program package ${ }^{34}$ and a comprehensive conformational search has been carried out. UV-Vis absorption spectra and Boltzmann weighted $\Delta \mathrm{E}_{S \mathrm{~T}}$ calculations (Tables S1S2) showed that different conformations did not exhibit significant differences in their excitation energies and on the ordering of the excited states. Hence, $\mathrm{T}_{1}$ geometry optimizations and related excited state calculations were carried out solely on the most stable conformer. The M06-2X functional 35 has been used together with the $6-31+G(d, p)$ basis set for $S_{0}$ and $T_{1}$ geometry optimizations. This choice is justified since M06-2X is well known to correctly reproduce long range interactions, electronic energies, and equilibrium geometries of compounds with aromatic ring systems. ${ }^{35}$ In order to increase the accuracy, $6-311++G(3 d f, 3 p d)^{36}$ and $6-311++G(2 d, 2 p)^{37}$ basis sets have been used for compounds including sulfur and phosphorus, respectively. Calculations have been performed taking into account the experimentally employed solvents, by the polarizable continuum model in the integral equation formalism (IEFPCM). CYLview software package has been used for visualization purposes. 38

Similar to ground state calculations, excited state calculations have been carried out by using Gaussian 16 software package. Tamm-Dancoff approximation (TDA) has been used, as this approach avoids problems related with triplet instability while maintaining a good description of the singlet excited states, hence yielding more accurate and balanced results. ${ }^{39,40} \mathrm{The}$ absorption spectra as well as the energies of the lowest lying singlet and triplet excited states have been computed with different functionals (B3LYP41, BLYP41, PBE042, M06-2X35) and 6$31+G(d, p)$ basis set, and the performances of these functionals have been evaluated with respect to experimental data. Absorption spectra have been modeled, including the effects of vibrational and thermal motion via Wigner Distribution sampling of the equilibrium region on the potential energy surface by generating 40 conformations via the Newton-X program ${ }^{43}$ on the equilibrium region of the PES.

NTOs and $\Phi_{S}$ indices have been calculated for the $S_{1}$ state by using the Gaussian 16 and Nancy_EX program packages, ${ }^{44}$ while hole and electron NTOs have been visualized with the Avogadro program package. $45 \Phi_{\mathrm{S}}$ index can be defined as the spatial overlap between attachment and detachment densities. Values closer to 1 indicate the presence of local excitation (LE) character, whereas values approaching 0 imply that the charge transfer (CT) character is dominant.44 Spin-orbit coupling (SOC) values between $S_{1}$ and $T_{1}$, and in some cases between $S_{1}$ and $T_{2}$, have been calculated using the Amsterdam Density Functional (ADF) software package by utilizing a DZP basis set. 46

\section{RESULTS AND DISCUSSION}

The benzophenone emitters investigated in this study have been grouped according to the type of acceptor moieties used. As depicted in Figure 3, Group 1 emitters are in the form of DA-D, they contain benzophenone acceptor cores (in red) bridged with various electron donating groups. They can be further subdivided into two subgroups: symmetric and asymmetric emitters. Symmetric emitters Px2BP12, DMAC-BP47, $\mathrm{Cz}_{2} \mathrm{BP}^{12}$ and CC2 $\mathrm{BP}^{12}$ contain phenoxazine (PXZ), dimethylacridine (DMAC) and carbazole (Cz) donors (in blue), respectively. Asymmetric emitters, A-BP-TA ${ }^{48}$ and OPDPO ${ }^{49}$ include donor groups such as thianthrene, phenothiazene (PTZ) and diphenylphosphineoxide.

Group 2 emitters have fused D-A ring structures where DBTBZ-PXZ ${ }^{50}$, DBT-BZ-PTZ ${ }^{25}$ and DBT-BZ-DMAC ${ }^{51}$ bear the acceptor unit dibenzothiophene-benzoyl (in red), while CP-BP-PXZ ${ }^{52}$ and CP-BP-DMAC52 have the acceptor unit of phenylcarbazolebenzoyl (in red). Donor groups such as PXZ, PTZ and DMAC have been employed in these emitters as well.

Group 3 emitters have para-substituted structures in which the acceptor unit is anthraquinone. ${ }^{18}$ These include D-A-D type emitters a1-a4 in which the donor units are diphenylamine (DPA), bis(4-biphenyl)amine, 3,6-di-tert-butylcarbazole and dimethylacridine (DMAC), respectively. Similarly, in the D- $\pi-A$ $\pi$-D structures, b1 and b4, the donor groups are DPA and DMAC.

Group 4 emitters have D-A type of structures in which the acceptor unit is xanthone. DMAC, PXZ and PTZ donors have been used in ACRXTN ${ }^{23}$, 3-PXZ-XO53 and PTZ-XT54, respectively. In MCz-XT21, 1,3,6,8-tetramethylcarbazole is present as the donor unit.

Finally, 7 non-TADF compounds have been selected from literature in an attempt to elucidate the main structural differences leading to TADF emission (Figure 4). While some of these nonTADF emitters have D-A type structures, including $M C 255$, OPM 56 and p-Cz ${ }^{57}$, others (ODFRCZ ${ }^{58}$, ODBTCZ ${ }^{59}, \mathrm{C}^{60}$ and $\mathrm{C}^{60}$ ) possess D-A-D type of structures.

\section{Benchmark Calculations and UV-Vis Absorption Spectra}

A series of widely used DFT functionals have been chosen for excited state calculations, BLYP, B3LYP, PBE0 and M06-2X. More specifically, UV-Vis absorption spectra have been generated for a group of emitters, and the results have been compared with the available experimental spectra.

The conformational space has been taken into account in the modeling of four compounds (Px2BP, Cz2BP, DBT-BZ-PXZ, DBT-BZ-DMAC) (Table S1). Their absorption spectra have been obtained as the union of the spectra of all the conformers according to their Boltzmann weights. The merged spectra have then been compared to those obtained from the most stable conformation only. Table S1 clearly demonstrates that weighted conformations exhibit similar photophysical features and spectral shapes as the most stable conformation: the maximum deviation being around $3 \mathrm{~nm}$ for B3LYP (DBT-BZ-PXZ), 4 $\mathrm{nm}$ for PBE0 (DBT-BZ-PXZ), and $2 \mathrm{~nm}$ for M06-2X (Cz2BP). In addition to the UV-Vis absorption spectra, different conformations of $\mathrm{Cz} 2 \mathrm{BP}$ and CC2BP have very similar $\Delta \mathrm{E}_{\text {ST }}$ values, the Boltzmann weighted $\triangle \mathrm{E}$ sT values of Cz2BP and CC2BP were found to be in good agreement with the experimental findings (Table S2). Thus, henceforth, the most stable conformer will solely be considered. In Tables S3-S5, the absorption spectra for Group 1 emitters and three emitters from Group 2 have been computed by using BLYP, B3LYP, PBE0 and M06-2X. The results suggest that the spectra obtained with M06-2X exhibit hypsochromic shift as compared to the experimental spectrum. 


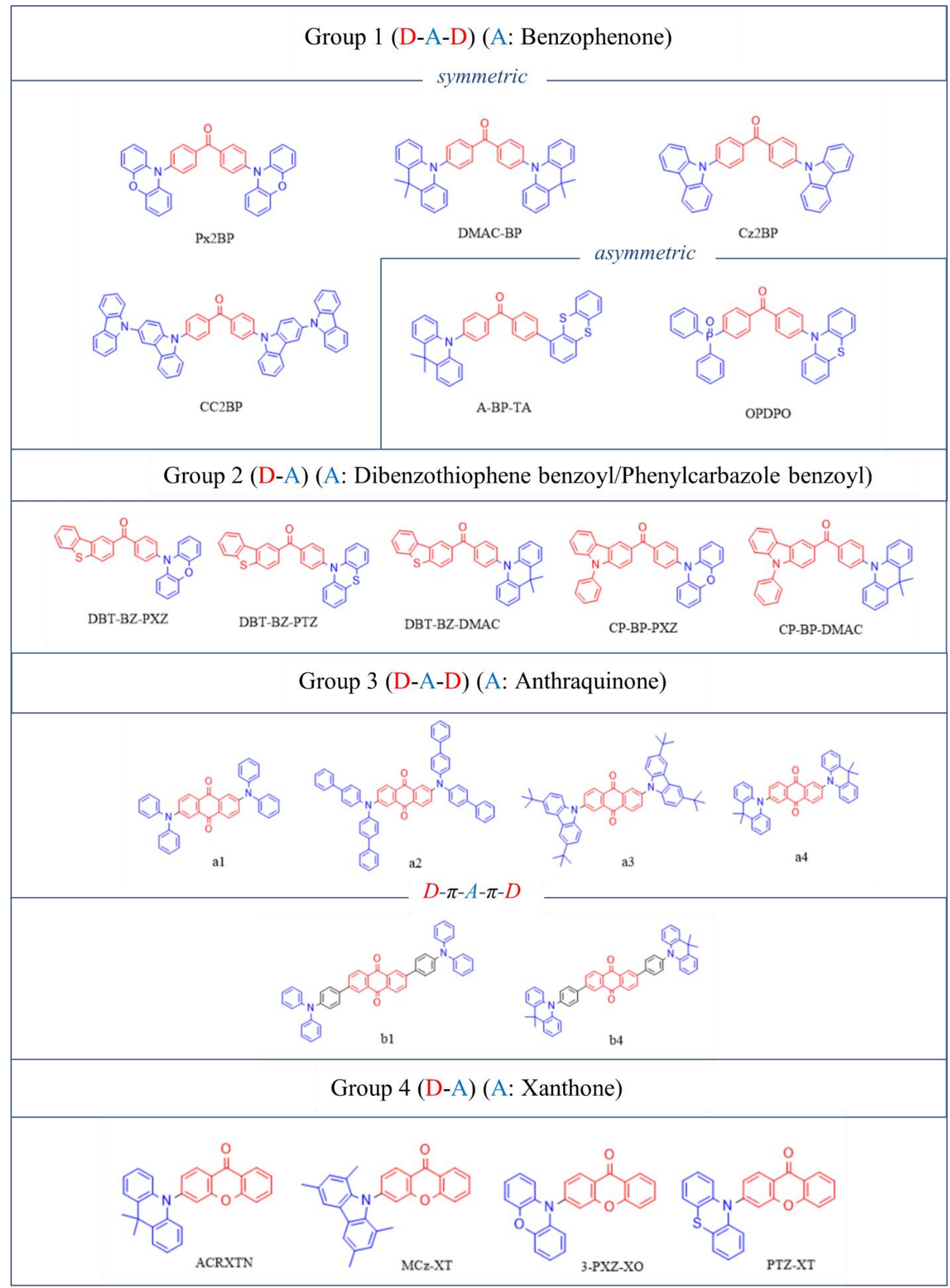

Figure 3. Classification of TADF emitters modeled in this study. 

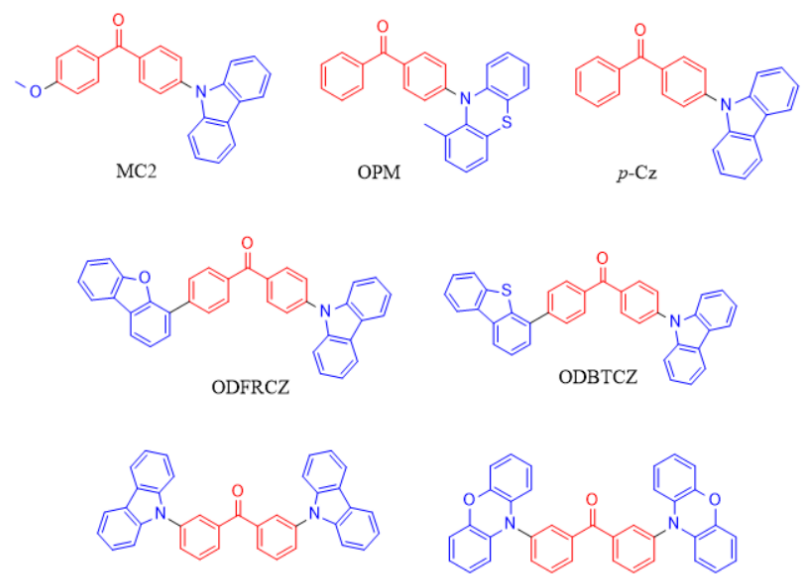

$\mathrm{C} 1$

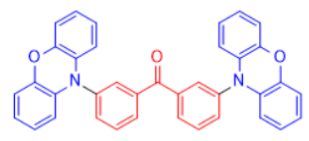

$\mathrm{C} 2$

Figure 4. Non-TADF benzophenone emitters.

As opposed to M06-2X, BLYP produced bathochromic shifts and lower $\Delta \mathrm{EsT}_{\mathrm{S}}$ values (energies are given in Tables S6-S7, histogram charts are depicted in Figures S1-S2) when compared with the experimental data. This behavior is most probably due to well-known unphysical stabilization of CT states by LDA functionals. ${ }^{61}$ In fact, hybrid B3LYP and PBE0 functionals yield the best agreement with experimental spectra and $\Delta \mathrm{E}_{\text {ST. Note }}$ also that BLYP also gave results consistent with experiment for a few molecules (Px2BP, DMAC-BP). This is probably due to the fact that, due to the relatively small size of the compounds, the CT states are not long-range and hence can be correctly reproduced by hybrid functionals as already observed for instance in some organometallic compounds, hence outperforming longrange corrected functionals. ${ }^{62}$

The absorption spectra of all other molecules have hence been calculated with BLYP, B3LYP and PBE0 functionals (Figures S3S7). From the results, it can be deduced that compounds with more rigid electron donating groups such as PXZ, PTZ and DMAC, possess a broadened band ( $350-500 \mathrm{~nm})$ appearing after the high intensity LE band $(\sim 300 \mathrm{~nm})$, which can be ascribed to the presence of CT states. ${ }^{63-65}$ Nevertheless, the reasonably rigid emitters of Group 4 also have blue-shifted absorption spectra, which is most likely due to the less efficient nature of the specific D-A type architecture. Indeed, the presence of a unique donor unit might be decreasing the CT character of the electronic transitions, coherently reduced $\mathrm{T}_{1}-\mathrm{S}_{1}$ upconversion efficiency has been previously reported for some DA type emitters. 66

\section{Donor-Acceptor Torsion Angles}

Several computational TADF descriptors were assessed in order to analyze and investigate the structural and photophysical phenomena related to TADF emissions. One of the most critical TADF descriptors is the torsion angle between the donor and acceptor units. The torsion angle must be close to $90^{\circ}$ degrees in an ideal TADF emitter, since an effective charge transfer configuration can be achieved by spatially separating the hole and electron densities and by breaking the conjugation pattern. ${ }^{67}$

The $\mathrm{S}_{0}$ and $\mathrm{T}_{1}$ optimized geometries of all emitters and the conformation energies (M06-2X/6-31+G(d,p)) are given in Supporting Information (Tables S8-S12). Figure 5 demonstrates the torsion angles measured for all emitters investigated in this study. Since symmetrical emitters have similar torsion angles for each donor-acceptor bond, only one of the torsion angles has been shown in Figure 5, while numerical values for torsion angles depicted in Figures S8-S12 are reported in Table S13. For asymmetrical emitters, the torsion angle of the electron donating unit has been ascribed based on the NTO analysis discussed in the following sections. Similarly, if the electron-hole density is localized on a different electron donating unit in the $\mathrm{T}_{1}$ equilibrium geometry, the corresponding torsion angle has been considered for the analysis of the $\mathrm{T}_{1}$ equilibrium geometries.

It is evident that the emitters containing rigid electron donating moieties, including DMAC, PXZ and PTZ, have significantly higher donor-acceptor torsion angles. While reasonably high

Opt: $M 06-2 X / 6-31+G(d, p)$

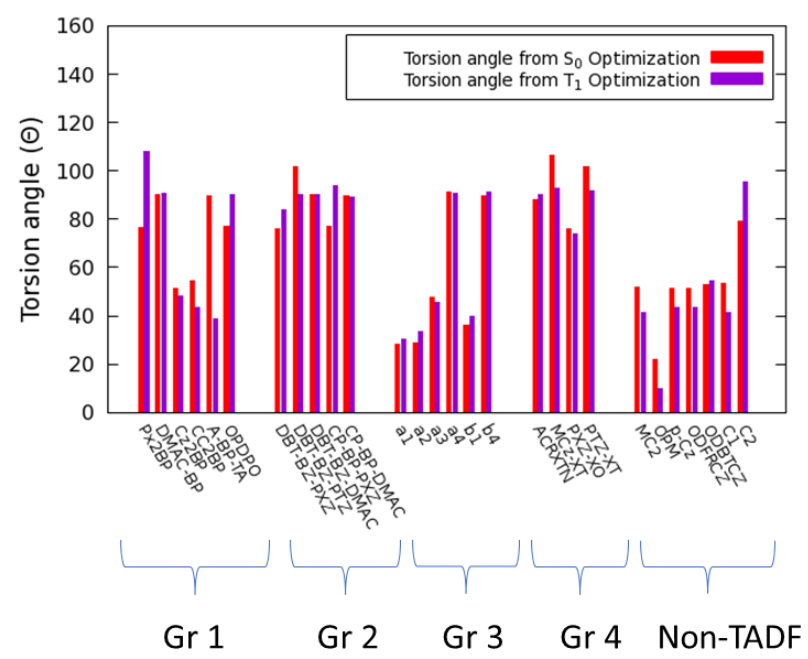

Figure 5. Donor-acceptor torsion angles for the $\mathrm{S}_{0}$ and $\mathrm{T}_{1}$ optimized geometries (M06-2X/6-31+G(d,p)) of the investigated emitters shown with stacked histograms.

torsion angles have been observed for Group 1, Group 2 and Group 4, Group 3 emitters bear relatively low torsion angles approximately in the range of $30^{\circ}-35^{\circ}$. This feature can be due to the presence of less rigid DPA donors whose rotation may lead to highly planar structure with extended $\pi$-conjugation patterns. The highest torsion angles belong to $\mathrm{a} 4 \mathrm{and} \mathrm{b} 4$ among Group 3 emitters due to the presence of DMAC electron donors in these groups. Indeed, the additional dimethyl units in the acridine structure impose sterical constraints that restrict the free rotation of the donor. Carbazole $(\mathrm{Cz})$ containing compounds also exhibit low torsion angles, around $55^{\circ}$, which are slightly higher than for the DPA-bearing compounds. Interestingly, the majority of the non-TADF compounds, where the donor unit is $\mathrm{Cz}$, have notably lower torsion angles, along with two compounds from Group 1 emitters (Cz2BP and CC2BP).

The torsion angles measured for Group 4 emitters are satisfactorily high and close to $90^{\circ}$ regardless of the electron donating unit. This situation can be attributed to the rigid xanthone skeleton that fixes the donor-acceptor torsion angle at a desirable position. This observation is in line with the study of Kreiza et al.17 Nonetheless, the obtained results suggest that such descriptor can only provide a partial understanding of TADF efficiency, since Group 3 compounds, which are known as TADF emitters, possess very low torsion angles, despite the presence of the rigid anthraquinone acceptor. Hence, a finer analysis of 
the property and reorganization of the electronic density should be considered to improve TADF efficiency predictions.

While in most cases the torsion angles are similar for both $\mathrm{T}_{1}$ and $\mathrm{S}_{0}$ equilibrium geometries, there is a sharp decrease in the torsion angle for $\mathrm{T}_{1}$ equilibrium geometry in A-BP-TA. Indeed, the hole density in the triplet state is localized on the less rigid electron donor thianthrene, causing a rather important planarization of the global structure. This will be better understood in the following section.

\section{Natural Transition Orbitals and $\Phi_{\text {s }}$ Indices}

Natural transition orbitals (NTOs) and $\Phi_{\mathrm{s}}$ indices have been calculated for all emitters in an attempt to determine the electron-hole density reorganization. Accordingly, electron and hole NTOs describing electronic density reorganization in the $\mathrm{S}_{1}$ state are shown in Tables S14-S23. Only the NTOs generated from B3LYP/6-31+G(d,p) densities are given since no remarkable difference has been observed with BLYP and PBE0. $\Phi_{\mathrm{S}}$ values describing the spatial overlap between attachment and detachment densities ${ }^{68}$ are also reported in Tables S24-S28.

In general, the overlap between electron and hole densities has been observed to be high for compounds bearing low D-A torsion angles. This is expected, since low torsion angles cause high degrees of $\pi$-delocalization and a corresponding planarization of the molecular core. As a result, compounds bearing $\mathrm{Cz}$ and DPA donors, including Cz2BP, CC2BP, a1, a2, a3, b1 and most of the non-TADF compounds, show highly overlapping hole and electron NTOs. Interestingly, in non-TADF emitters ODBTCZ and ODFRCZ, the highly flexible dibenzothiophene and dibenzofuran units did not show electron donating ability in $\mathrm{S}_{0}$ or $\mathrm{T}_{1}$ equilibrium geometries. Obviously, since high $\Phi_{\mathrm{s}}$ indices are indicative of spatially overlapping densities the corresponding excited states have a more prominent LE character. On the other hand, emitters with more rigid donor units (DMAC, PXZ and PTZ) usually have low amounts of overlap between their NTOs, and consequently small $\Phi_{\mathrm{S}}$ values, even in the presence of small torsion angles. Hence, even if more computationally expensive, the explicit analysis of the excited state density is much more informative in predicting TADF potential.

It is also noteworthy that the structural reorganization may have an undeniable impact on the amount of charge transfer from the accessible excited states, as in the case of excited state twisting. ${ }^{69}$ Indeed, some compounds, particularly those, containing butterfly-shaped PXZ, PTZ or thianthrene units, underwent drastic changes, more specifically, $\mathrm{T}_{1}$ equilibrium geometries exhibited planarization in the butterfly-shaped moieties (Table 1). This is, in turn, related to a change in the shape of the NTOs obtained from the $\mathrm{T}_{1}$ equilibrium geometries. The change in the NTO distribution is indicative of the fact that geometric relaxation leads, for some compounds, such as Px2BP, A-BP-TA, and $b 1$, to the population of a different diabatic state.

The most obvious change in the NTO localization pattern is seen in A-BP-TA (Figure 6), where at $\mathrm{T}_{1}$ equilibrium geometry, the hole NTO is localized mostly on the now planar thianthrene unit instead of the rigid DMAC unit. This effect also produces an increase in the $\Phi_{\mathrm{S}}$ indices at the $\mathrm{T}_{1}$ equilibrium geometry for Px2BP and its meta-substituted analogue C2 (Table S14 and Table S23). The electron NTO localizes only on one of the PXZ units, which becomes planar at $\mathrm{T}_{1}$ equilibrium geometry, this feature is also indicative of a consistent pattern, which is ob- served in two other similar cases. In the $\pi$-bridged anthraquinone-based b1 and b4, similar changes have been observed in the transition from $\mathrm{S}_{0}$ to $\mathrm{T}_{1}$ equilibrium geometry (Table S19). These consist of slight rotations in the bridging phenyl links, which cause disruptions in orthogonality and give rise to an increased electron delocalization. For a better visualization, the hole and electron NTOs and $\Phi_{\mathrm{s}}$ indices, calculated at $\mathrm{T}_{1}$ and $\mathrm{S}_{0}$ equilibrium geometries for two TADF and one non-TADF compounds are shown in Figure 6.

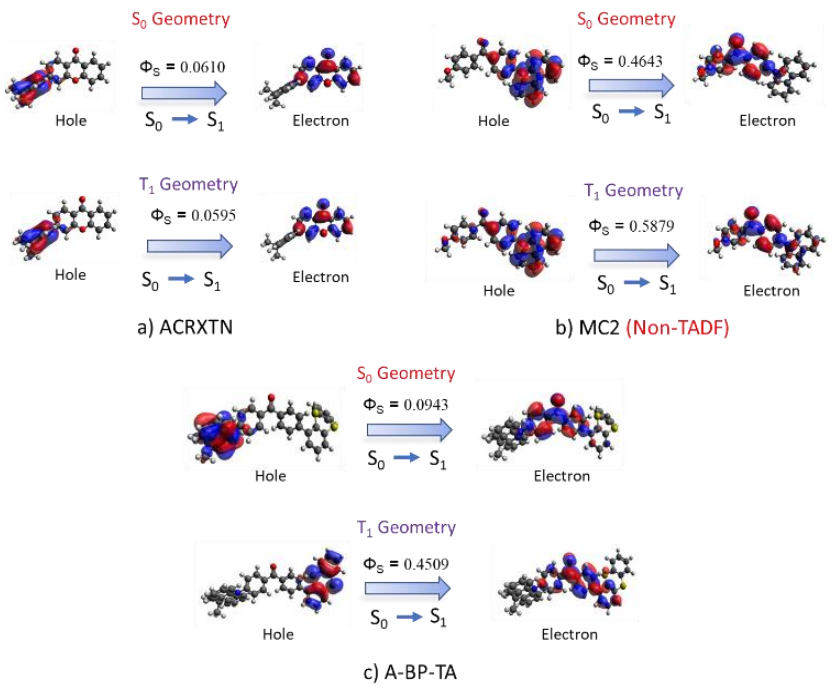

Figure 6. Hole-Electron levels and $\Phi_{\mathrm{S}}$ indices calculated with B3LYP $/ 6-31+G(d, p)$ for the molecules a) ACRXTN, b) MC2, c) A-BP-TA.

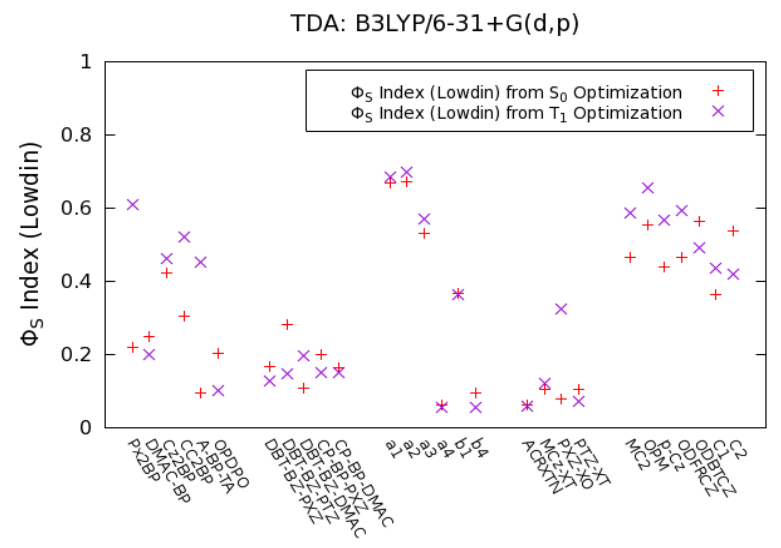

Figure 7. $\Phi_{\mathrm{s}}$ indices for the TADF emitters calculated from $\mathrm{S}_{0}$ and $\mathrm{T}_{1}$ geometries with B3LYP/6-31+G(d,p).

As expected, non-TADF compounds exhibit higher $\Phi_{\mathrm{S}}$, which are due to the presence of weaker electron donors like $\mathrm{Cz}$, dibenzofuran and dibenzothiophene. However, despite the fact that the only noticeable structural difference is related to the position of the PXZ substituents, the meta-substituted nonTADF $C 2$ also exhibits higher $\Phi_{\mathrm{S}}$ indices compared to its parasubstituted analogue, Px2BP, in line with its classification as a non-TADF compound. $\Phi_{s}$ indices calculated with B3LYP for all compounds are depicted in Figure 7 , and the $\Phi_{\mathrm{S}}$ indices calculated with PBE0 and BLYP are given in Figures S13-S14. TADF behavior is clearly inferred from these values, hence making this descriptor quite promising for the discrimination and prediction of optical properties with TADF potential. 
Table 1. $\mathrm{S}_{0}$ and $\mathrm{T}_{1}$ geometries of selected emitters with butterfly shaped electron donors.

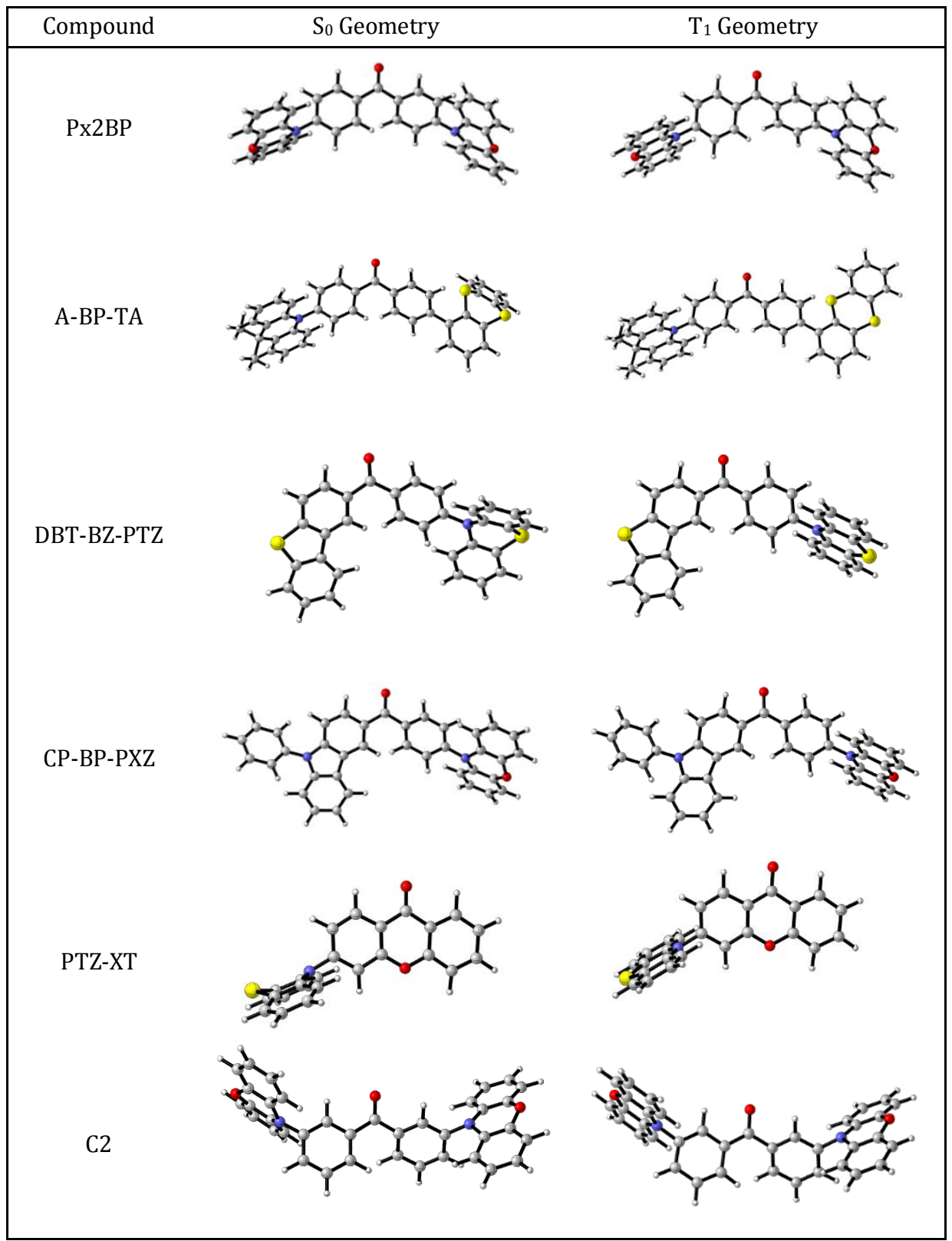

\section{Singlet-Triplet Energy Gaps ( $\Delta \mathrm{EsT})$}

The energy difference between the first singlet and triplet excited states is an important descriptor for predicting the possibility of TADF emission since the up-conversion of $\mathrm{T}_{1}$ to $\mathrm{S}_{1}$ can be achieved only if the energy gap is low enough to be overcome by thermal energy. According to previous studies, $\Delta \mathrm{E}_{\text {sт }}$ values above $0.3 \mathrm{eV}$ usually decrease the likelihood of the RISC process, whereas the up-conversion of the triplet becomes more likely if the $\Delta \mathrm{EsT}$ value is below $0.1 \mathrm{eV} .{ }^{70}$

Even though the RISC process usually takes place from the $\mathrm{T}_{1}$ state, upper lying triplet excited states may also influence the efficiency of RISC if they are in close proximity to $\mathrm{T}_{1}$. Spin-vi bronic coupling may cause state mixing between triplet energy levels, giving rise to strongly coupled $\mathrm{S}_{1}$ and $\mathrm{T}_{1}$ states. Therefore, the strong impact of internal conversion (IC) on ISC and RISC processes cannot be underestimated. ${ }^{71}$ Thus, the energy differences between $\mathrm{S}_{1}-\mathrm{T}_{2}\left(\Delta \mathrm{E}_{\mathrm{s} 1-\mathrm{T} 2}\right)$ states have also been reported along with the $\Delta \mathrm{E}_{\mathrm{S} 1-\mathrm{T} 1}$ values when the $\mathrm{T}_{2}$ state lies below $S_{1}$ for any given compound. The calculated values together with the experimental findings are reported in $\mathrm{Ta}$ bles S29-S33 and in Figure 8. 
TDA: B3LYP/6-31+G(d,p)

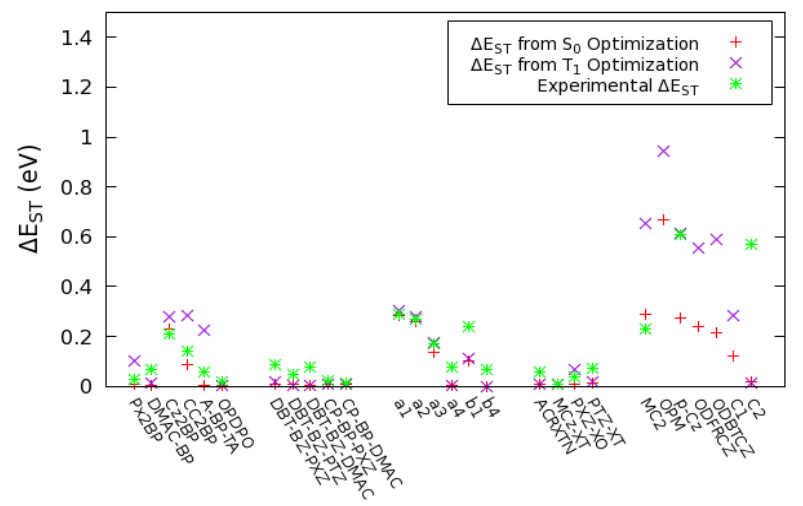

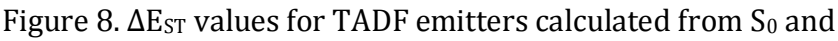
$\mathrm{T}_{1}$ geometries with B3LYP/6-31+G(d,p). (Experimental $\Delta \mathrm{E}_{S \mathrm{~T}}$ values also included)

Figure 8 depicts the $\Delta \mathrm{E}_{\mathrm{S} 1-\mathrm{T} 1}$ values calculated with B3LYP for almost all groups except for Group 3, since $\Delta \mathrm{E}_{\text {S1-T2 }}$ values calculated for them are in a better agreement with the experimental findings. It is evident that the lowest $\Delta \mathrm{E}_{\text {sт }}$ values have been obtained from the BLYP calculations, which is consistent with the lowest $\Phi_{\mathrm{S}}$ indices computed with this functional. On the other hand, B3LYP and PBE0 produced $\triangle$ EsT values closer to the experimental ones. Overall, the calculated $\Delta \mathrm{E}_{\text {ST }}$ values are usually consistent with the experimental observation, especially for the $\mathrm{S}_{0}$ geometries. The $\Delta \mathrm{E}_{\mathrm{sT}}$ values calculated with PBE0 and BLYP are also given in Figures S15-S16.

It is noteworthy that $\Delta \mathrm{E}_{\mathrm{ST}}$ values are closely correlated with torsion angles and $\Phi_{S}$ indices since the compounds possessing low torsion angles and high $\Phi_{\mathrm{s}}$ indices, such as Cz2BP, CC2BP, most of the Group 3 compounds and non-TADF compounds, also have relatively higher $\Delta \mathrm{E}_{\mathrm{S} 1-\mathrm{T} 2}$, and in some cases even high $\Delta \mathrm{E}_{\mathrm{S} 1-\mathrm{T} 1}$ values. Freely rotating donors DPA and sterically less hindered donors $\mathrm{Cz}$ lead to higher $\Delta$ Esт values because of enhanced geometrical relaxation. Despite having DPA donors, b1 has been observed to possess relatively low $\Delta \mathrm{E}_{\text {ST }}$ values, which may have been acquired through the phenyl bridges that lead to a higher spatial separation of electron and hole densities.

As expected, emitters with DMAC, PXZ or PTZ donors have satisfying $\Delta \mathrm{E}_{\text {ST }}$ values usually at both $\mathrm{S}_{0}$ and $\mathrm{T}_{1}$ equilibrium geometries due to the induced orthogonality. There is a sharp increase in the $\Delta \mathrm{E}_{\text {ST }}$ values obtained from the $\mathrm{T}_{1}$ equilibrium geometry of A-BP-TA, where the electron donating group is the less rigid thianthrene moiety. This is also consistent with the NTO calculations previously mentioned, pointing out the close relationship between $\Delta \mathrm{E}_{\text {sт }}$ and electron-hole separation. This result for A-BP-TA also further proves the reliability of NTO calculations and $\Phi_{\mathrm{S}}$ index in elucidating the excited state properties by relating them to the molecular structure.

Almost all of the non-TADF compounds have high $\Delta \mathrm{E}_{\text {ST }}$ values due to higher amounts of density overlap introduced by freely rotating donor-acceptor moieties, which planarize to enhance conjugation. However, C2 is an exception as it exhibits notably lower $\Delta \mathrm{EST}_{\mathrm{T}}$ values in spite of its high $\Phi_{\mathrm{S}}$ index and overlapping NTOs. The calculated $\Delta \mathrm{E}_{\text {ST }}$ value is also lower as compared with its experimental $\Delta \mathrm{E}_{S \mathrm{ST}}$ value, clearly identifying this compound as an outlier. The high experimental $\Delta \mathrm{E}_{\mathrm{s}}$ value can be explained by some side processes, such as oxygen quenching that increases the amount of non-radiative decays in solution. ${ }^{72}$ Still, out of 28 benzophenone derivatives investigated in this study, C2 is the only emitter in which an inverse relationship between $\Delta \mathrm{E}_{\text {ST }}$ values and $\Phi_{\mathrm{S}}$ indices was observed, and this can also be resulting from the insufficiency of our methodology in reproducing excited state energies for this compound.

\section{Spin-Orbit Couplings (SOC)}

The last descriptor analyzed in this study is the spin-orbit coupling (SOC). The mixing of the wavefunctions of singlet and triplet energy levels are indeed strongly dictated by SOC. 73 Therefore, by performing SOC calculations, crucial information regarding the feasibility of ISC or RISC processes is obtained. Indeed, SOC matrix elements significantly different from zero are necessary to attain RISC and, hence, TADF emission. However, SOC usually increases with atomic number (Z), thus small organic chromophores are usually less prone to ISC and RISC as compared to heavy-metal containing complexes. ${ }^{74}$ Additionally, the coupling of CT states produce negligible SOC values as a consequence of El-Sayed's rule, which highlights the need for local triplet excited states for an effective RISC process. ${ }^{73}$ Efficient TADF emitters usually have lower SOC values due to their orthogonal D-A type molecular backbones, where CT character is more pronounced, this unfavorable factor is, however, compensated by small singlet-triplet energy gaps, which are inversely proportional to the (R)ISC probability. Hence, SOC between the $\mathrm{S}_{1}$ and $\mathrm{T}_{1}$ states of TADF emitters could also indicate the presence of CT states. Since we are dealing with RISC, calculations were solely performed on equilibrium $\mathrm{T}_{1}$ geometries. SOC between $S_{1}$ and $T_{2}$ states have also been computed from the $\mathrm{T}_{1}$ geometries if the $\mathrm{T}_{2}$ lies below the $\mathrm{S} 1$ state, in a similar approach adopted for $\Delta \mathrm{E}_{\mathrm{ST}}$ calculations. The calculated SOC values have been reported in Tables S34-S38. For a better comprehension, the SOC values calculated with B3LYP, BLYP and PBE0 functionals have been displayed in Figure 9.

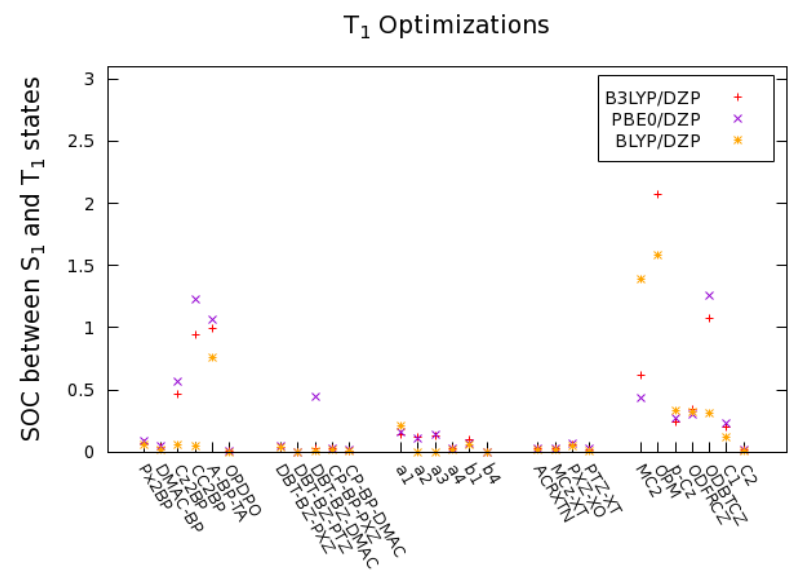

Figure 9. SOC values for TADF emitters calculated with B3LYP, BLYP and PBE0 using the $\mathrm{T}_{1}$ geometries.

The results indicate that the compounds possessing lower $\Delta \mathrm{E}_{\text {ST }}$

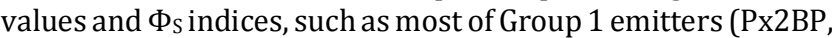
DMAC-BP, OPDPO), Group 2 emitters, a4, b4 and Group 4 emitters, exhibit extremely low SOC values as a result of the higher CT character of their $\mathrm{S}_{1}$ and $\mathrm{T}_{1}$ geometries. 
Higher SOC values have been obtained for compounds with less steric hindrance, including Cz2BP, CC2BP, most of Group 3 emitters, and all non-TADF emitters since local excitations become more favorable in these emitters.

A-BP-TA also exhibits relatively high SOC values, since its hole density becomes localized on the flexible thianthrene unit in its $\mathrm{T}_{1}$ geometry, reducing the $\mathrm{CT}$ character in a similar manner observed in its $\Delta \mathrm{EsT}_{\mathrm{T}}$ values and $\Phi_{\mathrm{s}}$ index. Additionally, higher SOC constants obtained for $\mathrm{S}_{1}-\mathrm{T}_{2}$ interactions may indicate that the triplet up-conversion might be taking place from the $\mathrm{T}_{2}$ rather than the $\mathrm{T}_{1}$ state.

Table 2 summarizes the D-A torsion angle, $\Phi_{\mathrm{S}}$ index, $\Delta \mathrm{E}_{\mathrm{S}} \mathrm{a}$ and SOC results of the TADF and non-TADF emitters investigated, showing a consistent behavior for all descriptors studied, pointing towards the successful accuracy of NTOs and $\Phi_{s}$ in inferring the TADF behavior. More importantly, in most cases the TADF capability may be correctly predicted by calculating the excited state indicators at the Franck-Condon geometry, hence avoiding rather expensive excited state optimizations.

Table 2. D-A torsion angles $\left({ }^{\circ}\right)$, $\Phi_{\text {S }}$ indices (Lowdin charge population), $\Delta \mathrm{EsT}_{\text {s }}$ values $(\mathrm{eV})$ and SOC constants for the TADF and nonTADF emitters calculated from the $\mathrm{T}_{1}$ geometries. (TDA: B3LYP $/ 6-31+G(d, p)$ )

\begin{tabular}{|c|c|c|c|c|c|}
\hline Group & Compound & Torsion Angle & $\Phi_{\mathrm{S}}$ Index & $\Delta \mathrm{E}_{\mathrm{ST}}$ & SOC \\
\hline \multirow{6}{*}{ Group 1} & Px2BP & 107.90 & 0.6097 & 0.104 & 0.073 \\
\hline & DMAC-BP & 90.91 & 0.1991 & 0.013 & 0.037 \\
\hline & Cz2BP & 48.19 & 0.4603 & 0.278 & 0.465 \\
\hline & CC2BP & 43.33 & 0.5206 & 0.284 & 0.942 \\
\hline & A-BP-TA & 38.90 & 0.4509 & 0.225 & 0.999 \\
\hline & OPDPO & 90.06 & 0.1009 & 0.004 & 0.004 \\
\hline \multirow{5}{*}{ Group 2} & DBT-BZ-PXZ & 83.84 & 0.1279 & 0.018 & 0.042 \\
\hline & DBT-BZ-PTZ & 90.21 & 0.1480 & 0.005 & 0.002 \\
\hline & DBT-BZ-DMAC & 90.11 & 0.1967 & 0.004 & 0.026 \\
\hline & CP-BP-PXZ & 93.80 & 0.1494 & 0.011 & 0.026 \\
\hline & CP-BP-DMAC & 88.90 & 0.1514 & 0.008 & 0.009 \\
\hline \multirow{6}{*}{ Group 3} & a1 & 30.54 & 0.6839 & 0.481 & 0.144 \\
\hline & a2 & 33.32 & 0.6992 & 0.428 & 0.120 \\
\hline & a3 & 45.42 & 0.5689 & 0.272 & 0.134 \\
\hline & a4 & 90.67 & 0.0546 & 0.007 & 0.025 \\
\hline & b1 & 39.68 & 0.3627 & 0.346 & 0.097 \\
\hline & b4 & 91.00 & 0.0544 & 0.001 & 0.000 \\
\hline \multirow{4}{*}{ Group 4} & ACRXTN & 90.21 & 0.0595 & 0.008 & 0.025 \\
\hline & MCz-XT & 92.89 & 0.1199 & 0.011 & 0.026 \\
\hline & 3-PXZ-XO & 74.04 & 0.3243 & 0.066 & 0.060 \\
\hline & PTZ-XT & 91.49 & 0.0721 & 0.018 & 0.022 \\
\hline \multirow{7}{*}{ Non-TADF } & MC2 & 41.45 & 0.5879 & 0.651 & 0.617 \\
\hline & OPM & 21.76 & 0.6566 & 0.944 & 2.070 \\
\hline & $p-\mathrm{Cz}$ & 43.66 & 0.5675 & 0.613 & 0.246 \\
\hline & ODFRCZ & 43.69 & 0.5936 & 0.553 & 0.345 \\
\hline & ODBTCZ & 52.73 & 0.4911 & 0.591 & 1.078 \\
\hline & $\mathrm{C} 1$ & 41.19 & 0.4369 & 0.283 & 0.202 \\
\hline & $\mathrm{C} 2$ & 95.14 & 0.4208 & 0.016 & 0.015 \\
\hline
\end{tabular}




\section{CONCLUSIONS}

In this study, the photophysical and structural properties of 21 benzophenone based TADF emitters, and 7 benzophenone based non-TADF emitters have been investigated by using computational descriptors with the aim to elucidate the factors causing RISC. The main descriptors have been identified as torsion angle between donor and acceptor, NTOs, $\Phi_{\mathrm{S}}$ indices, $\Delta \mathrm{E}_{S T}$ values, and SOC constants.

It was observed that the orthogonality of the molecular backbone is an important factor for achieving a successful RISC process. The compounds adopting a rigid molecular structure where free rotations are more restricted tend to have lower $\Delta \mathrm{E}_{\text {ST }}$ values and $\Phi_{\mathrm{S}}$ indices due to increased ICT through well separated electron and hole densities. Conversely, the emitters possessing less rigid and more freely rotating D-A arrangements were observed to have higher $\Delta \mathrm{E}_{S \mathrm{~T}}$ values and $\Phi_{\mathrm{S}}$ indices due to increased LE character induced by high amounts of overlap between the hole and electron and enhanced $\pi$-delocalization. Although molecular rigidity relies on both the molecular structures of the donor and acceptor, it was shown that the role of donor units is more dominant, as freely rotating electron donors such as Cz, DPA, or thianthrene, disrupt the orthogonality of the compounds, which leads to, in most cases, lower torsion angles $\left(\sim 30^{\circ}-55^{\circ}\right)$. Whereas the more rigid electron donors, such as DMAC, PXZ or PTZ, enhance TADF efficiency through increased orthogonality and electron/hole separation. However, the acceptor rigidity must also be taken into account. The compounds presenting more rigid and planar benzophenone derivatives, such as xanthone, as the acceptor moiety, exhibit satisfying TADF characteristics, regardless of the electron donors. On the contrary, the emitters bearing benzophenone acceptor, in which the loose phenyl rings have more free rotation, exhibit less satisfying TADF properties. The presence of a $\pi-$ bridge may also favorably affect the TADF properties owing to the increased spatial separation of donor and acceptor units as observed in compounds b1 and b4. Moreover, SOC values were calculated to be lower for the TADF compounds when the presence of highly twisted D-A frameworks induces lower $\Delta \mathrm{E}_{\text {ST }}$ values due to enhanced hole/electron separation.

Excited state calculations were carried out using both $\mathrm{S}_{0}$ and $\mathrm{T}_{1}$ geometries since excited state processes, such as RISC, should take place from the $\mathrm{T}_{1}$ equilibrium geometry. In some cases, a significant geometrical relaxation was observed, in particular for the butterfly-shaped PXZ, PTZ or thianthrene units, which become planar in $\mathrm{T}_{1}$ geometry. In these cases, different indicators were obtained for calculations performed at $\mathrm{S}_{0}$ or $\mathrm{T}_{1}$ geometries. However, in general, the outcome of the photophysical properties can be correctly inferred by solely considering the Franck-Condon geometry.

In summary, this study gives a comprehensive outlook on the relationship between molecular structure and photophysical features of a series of benzophenone based TADF and nonTADF compounds, employing computational tools and several descriptors. It can be concluded that these descriptors, especially the topological $\Phi_{\mathrm{S}}$ index, can be safely used to investigate TADF properties of different classes of compounds, as they predict the experimental findings quite accurately.

\section{ASSOCIATED CONTENT}

Supporting Information

3D representations of the optimized geometries, investigation of the conformational effects, benchmark calculations, UV-Vis absorption spectra, investigated donor-acceptor torsion angles, hole and electron NTOs, $\Phi_{s}$ indices, low lying singlet-triplet energy gaps, spin-orbit coupling values, $\mathrm{S}_{0}$ and $\mathrm{T}_{1}$ cartesian coordinates of the most stable conformations optimized at M06-2X/6-31+G(d,p) level of theory.

\section{AUTHOR INFORMATION}

Corresponding Authors

Viktorya Aviyente (aviye@boun.edu.tr)

Saron Catak (saron.catak@boun.edu.tr)

\section{CONFLICTS OF INTEREST}

There are no conflicts to declare.

\section{ACKNOWLEDGMENTS}

The computations were mainly performed using TUBITAK ULAKBIM High Performance and Grid Computing Center (TRUBA) and LPCT local computing resources. We also acknowledge the support from TUBITAK (Project Number:118Z914).

\section{REFERENCES}

(1) Tang, C. W.; VanSlyke, S. A. Organic Electroluminescent Diodes. Appl. Phys. Lett. 1987, 51 (12), 913-915.

(2) Huang, Y.; Hsiang, E. L.; Deng, M. Y.; Wu, S. T. MiniLED, Micro-LED and OLED Displays: Present Status and Future Perspectives. Light-Sci. Appl. 2020, 9 (1), 1-16.

(3) Tanaka, H.; Shizu, K.; Miyazaki, H.; Adachi, C. Efficient Green Thermally Activated Delayed Fluorescence (TADF) from a Phenoxazine-Triphenyltriazine (PXZ-TRZ) Derivative. Chem. Commun. 2012, 48 (93), 11392-11394.

(4) Xiao, L.; Chen, Z.; Qu, B.; Luo, J.; Kong, S.; Gong, Q.; Kido, J. Recent Progresses on Materials for Electrophosphorescent Organic Light-Emitting Devices. Adv. Mater. 2011, 23 (8), 926952.

(5) Dias, F. B.; Bourdakos, K. N.; Jankus, V.; Moss, K. C.; Kamtekar, K. T.; Bhalla, V.; Santos, J.; Bryce, M. R.; Monkman, A. P. Triplet Harvesting with $100 \%$ Efficiency by Way of Thermally Activated Delayed Fluorescence in Charge Transfer OLED Emitters. Adv. Mater. 2013, 25 (27), 3707-3714.

(6) Zhang, Q.; Li, J.; Shizu, K.; Huang, S.; Hirata, S.; Miyazaki, H.; Adachi, C. Design of Efficient Thermally Activated Delayed Fluorescence Materials for Pure Blue Organic Light Emitting Diodes. J. Am. Chem. Soc. 2012, 134 (36), 14706-14709. 
(7) Endo, A.; Sato, K.; Yoshimura, K.; Kai, T.; Kawada, A.; Miyazaki, H.; Adachi, C. Efficient Up-Conversion of Triplet Excitons into a Singlet State and Its Application for Organic Light Emitting Diodes. Appl. Phys. Lett. 2011, 98 (8), 2009-2012.

(8) Masui, K.; Nakanotani, H.; Adachi, C. Analysis of Exciton Annihilation in High-Efficiency Sky-Blue Organic LightEmitting Diodes with Thermally Activated Delayed Fluorescence. Org. Electron. 2013, 14 (11), 2721-2726.

(9) Uoyama, H.; Goushi, K.; Shizu, K.; Nomura, H.; Adachi, C. Highly Efficient Organic Light-Emitting Diodes from Delayed Fluorescence. Nature 2012, 492 (7428), 234-238.

(10) Reineke, S.; Baldo, M. A. Room Temperature Triplet State Spectroscopy of Organic Semiconductors. Sci. Rep. 2014, 4, 1-8.

(11) Marazzi, M.; Mai, S.; Roca-Sanjuán, D.; Delcey, M. G.; Lindh, R.; González, L.; Monari, A. Benzophenone Ultrafast Triplet Population: Revisiting the Kinetic Model by Surface-Hopping Dynamics. J. Phys. Chem. Lett. 2016, 7 (4), 622-626.

(12) Lee, S. Y.; Yasuda, T.; Yang, Y. S.; Zhang, Q.; Adachi, C. Luminous Butterflies: Efficient Exciton Harvesting by Benzophenone Derivatives for Full-Color Delayed Fluorescence OLEDs. Angew. Chem. Int. Ed. 2014, 53 (25), 6402-6406.

(13) Aloïse, S.; Ruckebusch, C.; Blanchet, L.; Réhault, J.; Buntinx, G.; Huvenne, J.P. The Benzophenone S1 $\left(n, \pi^{*}\right) \rightarrow \mathrm{T} 1$ $\left(n, \pi^{*}\right)$ States Intersystem Crossing Reinvestigated by Ultrafast Absorption Spectroscopy and Multivariate Curve Resolution. J. Phys. Chem A 2008, 112(2), 224-231.

(14) Huang, B.; Ban, X.; Sun, K.; Ma, Z.; Mei, Y.; Jiang, W.; Lin, B.; Sun, Y. Thermally Activated Delayed Fluorescence Materials Based on Benzophenone Derivative as Emitter for Efficient Solution-Processed Non-Doped Green OLED. Dyes Pigm. 2016, 133, 380-386.

(15) Keruckiene, R.; Keruckas, J.; Cekaviciute, M.; Volyniuk, D.; Lee, P. H.; Chiu, T. L.; Lee, J. H.; Grazulevicius, J. V. Meta-Substituted Benzophenones as Multifunctional Electroactive Materials for OLEDs. Dyes Pigm. 2020, 174, 108058.

(16) Gan, S.; Zhou, J.; Smith, T. A.; Su, H.; Luo, W.; Hong, Y.; Zhao, Z.; Tang, B. Z. New AIEgens with Delayed Fluorescence for Fluorescence Imaging and Fluorescence Lifetime Imaging of Living Cells. Mater. Chem. Front. 2017, 1, 2554-2558.

(17) Kreiza, G.; Banevičius, D.; Jovaišaitė, J.; Maleckaitė, K.; Gudeika, D.; Volyniuk, D.; Gražulevičius, J. V.; Juršènas, S.; Kazlauskas, K. Suppression of Benzophenone-Induced Triplet Quenching for Enhanced TADF Performance. J. Mater. Chem. C 2019, 7 (37), 11522-11531.

(18) Zhang, Q.; Kuwabara, H.; Potscavage, W. J.; Huang, S.; Hatae, Y.; Shibata, T.; Adachi, C. Anthraquinone-Based Intramolecular Charge-Transfer Compounds: Computational Molecular Design, Thermally Activated Delayed Fluorescence, and Highly Efficient Red Electroluminescence. J. Am. Chem. Soc. 2014, 136 (52), 18070-18081.
(19) Bin, H.; Ji, Y.; Li, Z.; Zhou, N.; Jiang, W.; Feng, Y.; Lin, B.; Sun, Y. Simple Aggregation-Induced Delayed Fluorescence Materials Based on Anthraquinone Derivatives for Highly Efficient Solution-Processed Red OLEDs. J. Lumin. 2017, 187, 414-420.

(20) Zhang, Y.; Ma, H.; Wang, S.; Li, Z.; Ye, K.; Zhang, J.; Liu, Y.; Peng, Q.; Wang, Y. Supramolecular Structure-Dependent Thermally-Activated Delayed Fluorescence (TADF) Properties of Organic Polymorphs. J. Phys. Chem. C 2016, 120 (35), 1975919767.

(21) Lee, J.; Aizawa, N.; Numata, M.; Adachi, C.; Yasuda, T. Versatile Molecular Functionalization for Inhibiting Concentration Quenching of Thermally Activated Delayed Fluorescence. Adv. Mater. 2017, 29 (4), 1-6.

(22) Aizawa, N.; Tsou, C. J.; Park, I. S.; Yasuda, T. Aggregation-Induced Delayed Fluorescence from Phenothiazine-Containing Donor-Acceptor Molecules for High-Efficiency NonDoped Organic Light-Emitting Diodes. Polym. J. 2017, 49 (1), 197-202.

(23) Nakanotani, H.; Higuchi, T.; Furukawa, T.; Masui, K.; Morimoto, K.; Numata, M.; Tanaka, H.; Sagara, Y.; Yasuda, T.; Adachi, C. High-Efficiency Organic Light-Emitting Diodes with Fluorescent Emitters. Nat. Commun. 2014, 5, 1-7.

(24) Guo, J.; Li, X. L.; Nie, H.; Luo, W.; Gan, S.; Hu, S.; Hu, R.; Qin, A.; Zhao, Z.; Su, S. J.; Tang, B. Z. Achieving High-Performance Nondoped OLEDs with Extremely Small Efficiency Roll-Off by Combining Aggregation-Induced Emission and Thermally Activated Delayed Fluorescence. Adv. Funct. Mater. 2017, 27 (13).

(25) Guo, J.; Li, X. L.; Nie, H.; Luo, W.; Hu, R.; Qin, A.; Zhao, Z.; Su, S. J.; Tang, B. Z. Robust Luminescent Materials with Prominent Aggregation-Induced Emission and Thermally Activated Delayed Fluorescence for High-Performance Organic LightEmitting Diodes. Chem. Mater. 2017, 29 (8), 3623-3631.

(26) Huang, J.; Nie, H.; Zeng, J.; Zhuang, Z.; Gan, S.; Cai, Y.; Guo, J.; Su, S. J.; Zhao, Z.; Tang, B. Z. Highly Efficient Nondoped OLEDs with Negligible Efficiency Roll-Off Fabricated from Aggregation-Induced Delayed Fluorescence Luminogens. Angew. Chem. Int. Ed. 2017, 56 (42), 12971-12976.

(27) Chen, Z.; Wu, Z.; Ni, F.; Zhong, C.; Zeng, W.; Wei, D.; An, K.; Ma, D.; Yang, C. Emitters with a Pyridine-3,5-Dicarbonitrile Core and Short Delayed Fluorescence Lifetimes of about 1.5 Ms: Orange-Red TADF-Based OLEDs with Very Slow Efficiency Roll-Offs at High Luminance. J. Mater. Chem. C 2018, 6 (24), 6543-6548.

(28) Olivier, Y.; Sancho-Garcia, J. C.; Muccioli, L.; D’Avino, G.; Beljonne, D. Computational Design of Thermally Activated Delayed Fluorescence Materials: The Challenges Ahead. J. Phys. Chem. Lett. 2018, 9 (20), 6149-6163.

(29) Mewes, J. M. Modeling TADF in Organic Emitters Requires a Careful Consideration of the Environment and Going beyond the Franck-Condon Approximation. Phys. Chem. Chem. Phys. 2018, 20 (18), 12454-12469.

(30) Kang, S.; Jeon, S. H.; Cho, Y. M.; Kim, Y. J.; Kim, T.; Lee, J. Y. The Key Role of Acceptor Moieties on the Structural and the 
Electronic Properties of Thermally Activated Delayed Fluorescence Emitters in Excited States: A Computational Study. Org. Electron. 2020, 78, 105595.

(31) Sanz-Rodrigo, J.; Olivier, Y.; Sancho-García, J. C. Computational Studies of Molecular Materials for Unconventional Energy Conversion: The Challenge of Light Emission by Thermally Activated Delayed Fluorescence. Molecules 2020, 25, 112.

(32) Zhu, Q.; Guo, X.; Zhang, J. Theoretical Study on Photophysical Properties of a Series of Functional Pyrimidine-Based Organic Light-Emitting Diodes Emitters Presenting Thermally Activated Delayed Fluorescence. J. Comput. Chem. 2019, 40 (16), 1578-1585.

(33) Tavakoli, M.; Ahmadvand, H.; Alaei, M.; Ranjbari, M. A. Ab-Initio Search for Efficient Red Thermally Activated Delayed Fluorescence Molecules for Organic Light Emitting Diodes. Spectrochim. Acta A Mol. Biomol. Spectrosc. 2021, 246, 118952.

(34) Frisch G. W.; Schlegel, H. B.; Scuseria, G. E.; Robb, M. A.; Cheeseman, J. R.; Scalmani, G.; Barone, V.; Petersson, G. A.; Nakatsuji, H.; Li, X.; Caricato, M.; Marenich, A. V.; Bloino, J.; Janesko, B. G.; Gomperts, R.; Mennucci, B.; Hratch, D. J., M. J. ; T. Gaussian 16, Rev. A.03. Gaussian, Inc., Wallingford, CT 2016.

(35) Josa, D.; Rodríguez-Otero, J.; Cabaleiro-Lago, E. M.; Rellán-Piñeiro, M. Analysis of the Performance of DFT-D, M05$2 \mathrm{X}$ and M06-2X Functionals for Studying $\pi \cdots \pi$ Interactions. Chem. Phys. Lett. 2013, 557, 170-175.

(36) Kolleth, A.; Müller, S.; Lumbroso, A.; Tanriver, G.; Catak, S.; Sulzer-Mossé, S.; De Mesmaeker, A. Access to 3-Aminobenzothiophenes and 3-Aminothiophenes Fused to 5-Membered Heteroaromatic Rings through $6 \pi$-Electrocyclization Reaction of Keteniminium Salts. Tetrahedron Lett. 2018, 59 (34), 3242-3248.

(37) Zabaradsti, A.; Kakanejadifard, A.; Ghasemian, M. Theoretical Study of Molecular Interactions of Phosphorus Ylide with Hypohalous Acids $\mathrm{HOF}, \mathrm{HOCl}$ and $\mathrm{HOBr}$. Comput. Theor. Chem. 2012, 989, 1-6.

(38) Legault, C.Y. CYLview, 1.0b. Université de Sherbrooke 2009.

(39) Hirata, S.; Head-Gordon, M. Time-Dependent Density Functional Theory within the Tamm-Dancoff Approximation. Chem. Phys. Lett. 1999, 314 (3-4), 291-299.

(40) Chantzis, A.; Laurent, A. D.; Adamo, C.; Jacquemin, D. Is the Tamm-Dancoff Approximation Reliable for the Calculation of Absorption and Fluorescence Band Shapes? J. Chem. Theory Comput. 2013, 9 (10), 4517-4525.

(41) Liang, K.; Zheng, C.; Wang, K.; Liu, W.; Guo, Z.; Li, Y.; Zhang, X. Theoretical Investigation of the Singlet-Triplet Splittings for Carbazole-Based Thermally Activated Delayed Fluorescence Emitters. Phys. Chem. Chem. Phys. 2016, 18 (38), 26623-26629.

(42) Alipour, M.; Karimi, N. Dissecting the Accountability of Parameterized and Parameter-Free Single-Hybrid and Double-Hybrid Functionals for Photophysical Properties of TADFBased OLEDs. J. Chem. Phys. 2017, 146 (23).
(43) Barbatti, M.; Ruckenbauer, M.; Plasser, F.; Pittner, J.; Granucci, G.; Persico, M.; Lischka, H. Newton-X: A Surface-Hopping Program for Nonadiabatic Molecular Dynamics. Wiley Interdiscip. Rev. Comput. Mol. Sci. 2014, 4 (1), 26-33.

(44) Etienne, T.; Assfeld, X.; Monari, A. New Insight into the Topology of Excited States through Detachment/Attachment Density Matrices-Based Centroids of Charge. J. Chem. Theory Comput. 2014, 10 (9), 3906-3914.

(45) Hanwell, M. D.; Curtis, D. E.; Lonie, D. C.; Vandermeerschd, T.; Zurek, E.; Hutchison, G. R. Avogadro: An Advanced Semantic Chemical Editor, Visualization, and Analysis Platform. J. Cheminformatics 2012, 4 (1), 1-17.

(46) te Velde, G.; Bickelhaupt, F. M.; Baerends, E. J.; Fonseca Guerra, C.; van Gisbergen, S. J. A.; Snijders, J. G.; Ziegler, T. Chemistry with ADF. J. Comput. Chem. 2001, 22 (9), 931-967.

(47) Zhang, Q.; Tsang, D.; Kuwabara, H.; Hatae, Y.; Li, B.; Takahashi, T.; Lee, S. Y.; Yasuda, T.; Adachi, C. Nearly 100\% Internal Quantum Efficiency in Undoped Electroluminescent Devices Employing Pure Organic Emitters. Adv. Mater. 2015, 27 (12), 2096-2100.

(48) Tomkeviciene, A.; Matulaitis, T.; Guzauskas, M.; Andruleviciene, V.; Volyniuk, D.; Grazulevicius, J. V. Thianthrene and Acridan-Substituted Benzophenone or Diphenylsulfone: Effect of Triplet Harvesting via TADF and Phosphorescence on Efficiency of All-Organic OLEDS. Org. Electron. 2019, 70, 227239.

(49) Chen, X.; Yang, Z.; Xie, Z.; Zhao, J.; Yang, Z.; Zhang, Y.; Aldred, M. P.; Chi, Z. An Efficient Yellow Thermally Activated Delayed Fluorescence Emitter with Universal Applications in Both Doped and Non-Doped Organic Light-Emitting Diodes. Mater. Chem. Front. 2018, 2 (5), 1017-1023.

(50) Guo, J.; Li, X. L.; Nie, H.; Luo, W.; Hu, R.; Qin, A.; Zhao, Z.; Su, S. J.; Tang, B. Z. Robust Luminescent Materials with Prominent Aggregation-Induced Emission and Thermally Activated Delayed Fluorescence for High-Performance Organic LightEmitting Diodes. Chem. Mater. 2017, 29 (8), 3623-3631.

(51) Guo, J.; Li, X. L.; Nie, H.; Luo, W.; Gan, S.; Hu, S.; Hu, R.; Qin, A.; Zhao, Z.; Su, S. J.; Tang, B. Z. Achieving High-Performance Nondoped OLEDs with Extremely Small Efficiency Roll-Off by Combining Aggregation-Induced Emission and Thermally Activated Delayed Fluorescence. Adv. Funct. Mater. 2017, 27 (13).

(52) Huang, J.; Nie, H.; Zeng, J.; Zhuang, Z.; Gan, S.; Cai, Y.; Guo, J.; Su, S. J.; Zhao, Z.; Tang, B. Z. Highly Efficient Nondoped OLEDs with Negligible Efficiency Roll-Off Fabricated from Aggregation-Induced Delayed Fluorescence Luminogens. Angew. Chem. Int. Ed. 2017, 56 (42), 12971-12976.

(53) Zhang, Y.; Ma, H.; Wang, S.; Li, Z.; Ye, K.; Zhang, J.; Liu, Y.; Peng, Q.; Wang, Y. Supramolecular Structure-Dependent Thermally-Activated Delayed Fluorescence (TADF) Properties of Organic Polymorphs. J. Phys. Chem. C 2016, 120 (35), 1975919767.

(54) Lee, I. H.; Song, W.; Lee, J. Y. Aggregation-Induced Emission Type Thermally Activated Delayed Fluorescent Materials for High Efficiency in Non-Doped Organic Light-Emitting Diodes. Org. Electron. 2016, 29, 22-26. 
(55) Wei, Q.; Kleine, P.; Karpov, Y.; Qiu, X.; Komber, H.; Sahre, K.; Kiriy, A.; Lygaitis, R.; Lenk, S.; Reineke, S.; Voit, B. Conjugation-Induced Thermally Activated Delayed Fluorescence (TADF): From Conventional Non-TADF Units to TADF-Active Polymers. Adv. Funct. Mater. 2017, 27 (7), 1-11.

(56) Chen, C.; Huang, R.; Batsanov, A. S.; Pander, P.; Hsu, Y.T.; Chi, Z.; Dias, F. B.; Bryce, M. R. Intramolecular Charge Transfer Controls Switching Between Room Temperature Phosphorescence and Thermally Activated Delayed Fluorescence. Angew. Chem. 2018, 130 (50), 16645-16649.

(57) Mao, Z.; Yang, Z.; Xu, C.; Xie, Z.; Jiang, L.; Gu, F. L.; Zhao, J.; Zhang, Y.; Aldred, M. P.; Chi, Z. Two-Photon-Excited Ultralong Organic Room Temperature Phosphorescence by Dual-Channel Triplet Harvesting. Chem. Sci. 2019, 10 (31), 7352-7357.

(58) Huang, L.; Liu, L.; Li, X.; Hu, H.; Chen, M.; Yang, Q.; Ma, Z.; Jia, X. Crystal-State Photochromism and Dual-Mode Mechanochromism of an Organic Molecule with Fluorescence, RoomTemperature Phosphorescence, and Delayed Fluorescence. Angew. Chem. 2019, 131 (46), 16597-16602.

(59) Huang, L.; Liu, J.; Liu, L.; Yang, Q.; Ma, Z.; Jia, X. A D-AD’ Type Organic Molecule with Persistent Phosphorescence Exhibiting Dual-Mode Mechanochromism. Dyes Pigm. 2020, 173, 107963.

(60) Keruckiene, R.; Keruckas, J.; Cekaviciute, M.; Volyniuk, D.; Lee, P. H.; Chiu, T. L.; Lee, J. H.; Grazulevicius, J. V. Meta-Substituted Benzophenones as Multifunctional Electroactive Materials for OLEDs. Dyes Pigm. 2020, 174, 108058.

(61) Wanko, M.; García-Risueño, P.; Rubio, A. Excited States of the Green Fluorescent Protein Chromophore: Performance of Ab Initio and Semi-Empirical Methods. Phys. Status Solidi B Basic Res. 2012, 249 (2), 392-400.

(62) Very, T.; Despax, S.; Hébraud, P.; Monari, A.; Assfeld, X. Spectral Properties of Polypyridyl Ruthenium Complexes Intercalated in DNA: Theoretical Insights into the Surrounding Effects of [Ru(Dppz)(Bpy) 2]2+. Phys. Chem. Chem. Phys. 2012, 14 (36), 12496-12504.

(63) Laguitton-Pasquier, H.; Pansu, R.; Chauvet, J.-P.; Collet, A.; Faure, J.; Lapouyade, R. The Charge Transfer State of Excited Bianthryl and a Derivative: Solvatochromism, Emission CT Spectra Broadening in Homogeneous Solvents. Chem. Phys. 1996, 212 (2-3), 437-455.

(64) Banerjee, S.; Both, A. K.; Sarkar, M. Probing the Aggregation and Signaling Behavior of Some Twisted 9,9'-Bianthryl Derivatives: Observation of Aggregation-Induced Blue-Shifted Emission. ACS Omega 2018, 3 (11), 15709-15724.
(65) Numata, M.; Yasuda, T.; Adachi, C. High Efficiency Pure Blue Thermally Activated Delayed Fluorescence Molecules Having 10H-Phenoxaborin and Acridan Units. Chem. Commun. 2015, 51 (46), 9443-9446.

(66) Chen, X. K.; Zhang, S. F.; Fan, J. X.; Ren, A. M. Nature of Highly Efficient Thermally Activated Delayed Fluorescence in Organic Light-Emitting Diode Emitters: Nonadiabatic Effect between Excited States. J. Phys. Chem. C 2015, 119 (18), 97289733.

(67) Meng, G.; Chen, X.; Wang, X.; Wang, N.; Peng, T.; Wang, S. Isomeric Bright Sky-Blue TADF Emitters Based on Bisacridine Decorated DBNA: Impact of Donor Locations on Luminescent and Electroluminescent Properties. Adv. Opt. Mater. 2019, 7 (11), 1-14.

(68) Etienne, T.; Gattuso, H.; Michaux, C.; Monari, A.; Assfeld, X.; Perpète, E. A. Fluorene-Imidazole Dyes Excited States from First-Principles Calculations-Topological Insights. Theor. Chem. Acc. 2016, 135 (4), 1-11.

(69) Gulbinas, V.; Kodis, G.; Jursenas, S.; Valkunas, L.; Gruodis, A.; Mialocq, J. C.; Pommeret, S.; Gustavsson, T. Charge Transfer Induced Excited State Twisting of N,N-Dimethylaminobenzylidene-1,3-Indandione in Solution. J. Phys. Chem. A 1999, 103 (20), 3969-3980.

(70) Dias, F. B.; Bourdakos, K. N.; Jankus, V.; Moss, K. C.; Kamtekar, K. T.; Bhalla, V.; Santos, J.; Bryce, M. R.; Monkman, A. P. Triplet Harvesting with $100 \%$ Efficiency by Way of Thermally Activated Delayed Fluorescence in Charge Transfer OLED Emitters. Adv. Mater. 2013, 25 (27), 3707-3714.

(71) Huang, R.; Avó, J.; Northey, T.; Chaning-Pearce, E.; Dos Santos, P. L.; Ward, J. S.; Data, P.; Etherington, M. K.; Fox, M. A.; Penfold, T. J.; Berberan-Santos, M. N.; Lima, J. C.; Bryce, M. R.; Dias, F. B. The Contributions of Molecular Vibrations and Higher Triplet Levels to the Intersystem Crossing Mechanism in Metal-Free Organic Emitters. J. Mater. Chem. C 2017, 5 (25), 6269-6280.

(72) Notsuka, N.; Nakanotani, H.; Noda, H.; Goushi, K.; Adachi, C. Observation of Nonradiative Deactivation Behavior from Singlet and Triplet States of Thermally Activated Delayed Fluorescence Emitters in Solution. J. Phys. Chem. Lett. 2020, 11 (2), 562-566.

(73) Huang, T.; Jiang, W.; Duan, L. Recent Progress in Solution Processable TADF Materials for Organic Light-Emitting Diodes. J. Mater. Chem. C 2018, 6 (21), 5577-5596.

(74) Marian, C. M. Spin-Orbit Coupling and Intersystem Crossing in Molecules. Wiley Interdiscip. Rev. Comput. Mol. Sci 2012, 2 (2), 187-203. 
Table of Contents (TOC) Graphic

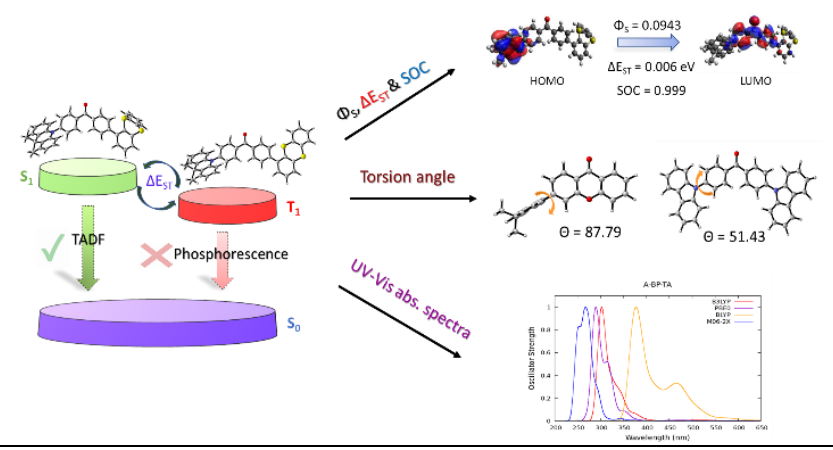

